\title{
A New Adaptive EDCA Approach to QoS of Wireless Communications
}

\author{
Huibin Wang, Yang Hu, Lili Zhang, Wei Xia \\ College of Computer and Information Engineering, Hohai University, Nanjing, China. \\ Email: hhuwhb@gmail.com
}

Received July $15^{\text {th }}, 2010$; revised July $19^{\text {th }}, 2010$; accepted July $25^{\text {th }}, 2010$.

\begin{abstract}
Under the background of intelligent transportation application, QoS for various services is different in wireless communication. Based on the MAC layer protocol, this paper analyzes the QoS in IEEE 802.11 MAC protocol framework, and proposes a new design of a Differentiation Enhanced Adaptive EDCA (enhanced distribution channel access) approach. The proposed approach adjusts the window zooming dynamically according to the collision rate in sending data frames, makes random offset, and further distinguishes the competition parameters of the data frames that have the same priority, so as to reduce the conflict among the data frames, and improve the channel utilization. Experiments with different service cases were conducted. The simulation results show that: comparing with the conventional EDCA method, the proposed approach can ensure that high priority services are sent with priority, and the overall QoS is highly improved.
\end{abstract}

Keywords: Intelligent Transportation Systems, Wireless Communi-Cation, Mac Layer Protocol, Enhanced Distribution Channel Access

\section{Introduction}

In the intelligent transportation systems (ITS), there are two wireless communication modes: car to car and car to road. They are the foundation of providing information exchange and service. Wireless communication improves the service active safety technology further. In early days, the main research topics of the intelligent transportation wireless communication technology are vehicles location and distance measurement between vehicles etc $[1,2]$ in collaborative driving application. In recent years, more and more researches focus on transmission of service information and mass data flows such as audio and video and so on. In researches and applications, the modern wireless communication technologies are adopted. For example, in Fleetnet-road network project (see http://www. et2.tu-harburg.de/fleetnet, Fleetnet) and CarTalk 2000 project (see http://www.cartalk2000.net, Cartalk 2000 web site), wireless LAN and cellular networks are employed.

In different intelligent transportation application scenarios, each service has stringent requirements for QoS of wireless communications. For example, in the active safety application scenarios, the delay of sending the se curity message requires to reach ms level. While in emergency voice communication application, packet loss rate of voice data stream can't exceed $1 \%$. QoS can be described in different aspects in the communication, such as the network throughput and delays. At present, the method of improving QoS generally uses separate-layer or crosslayer. Cross-layer program has the advantage of improving the efficiency of the protocol stack. But its network protocol is complex and is difficult to maintain. Therefore, in the application background, the main issue is separate-layer QoS program in recent days.

The MAC layer protocol controls node accessing to wireless channel and message transmission occupying wireless medium, so as to provide reliable data transfer for upper layer, and guarantee the overall performance of the network. Therefore, MAC layer is the focus of communication protocol in intelligent transportation application. For example, in the EDCA (enhanced distribution channel access) mechanism of the IEEE 802.11p [3] standard, the service data accesses to channel by a competitive way. And further distinction of priority-based QoS throu- gh distributing competitive parameters is achieved. CarTalk 2000 Project proposed the mechanism of Ad Hoc MAC [4]. It adopts dynamic TDMA mechanism, and each service accesses to the channel by making an appointment. The article [5] proposed the D-MAC mechanism, which reduces transmission conflict by using the directional 
antenna. Kaichi F. et al. proposed the VRCP mechanism [6], which can work in Central control or ad hoc network. And it can dynamically switch according to whether the node is in the coverage area of the central controller.

However, the existing programs for intelligent transportation cannot fully meet the requirements in real-world applications. For network throughput and delay, this paper lays special stress on analyzing the MAC layer, and emphatically studies the performance of QoS of EDCA mechanism in intelligent transportation and designs a new mechanism to improve it. Then simulate the algorithm and analyze the experiment results. This paper is structured as follows: Section 2 introduces the principle of EDCA mechanism and the support to QoS. Section 3 designs and describes a differentiation enhanced adaptive EDCA mechanism. Section 4 simulates on the OPNET platform and analyzes the results. Section 5 is the summary.

\section{Related Work}

The MAC layer provides three mechanisms and protocols in the intelligent transportation with wireless communication, which are described as follows:

1) CSMA/CD-based Protocol, such as the $802.11 \mathrm{p}$. CSMA/CD uses the mechanism of listen-before-talk (LBT). Each node in the system senses the channel before sending the packet. If the channel is idle, send data. If the channel is busy, continue to sense until it becomes idle, and then use binary backoff mechanism to reduce the probability of collision.

2) TDMA-based protocol, such as RR-ALOHA. Protocol, a channel is divided into several time slots (called BASIC CHANNEL, BC). Besides the payload of each channel, FI (Frame Information) is also transmitted. FI includes the idle state of each slot. Any terminal can ask for the establishment of $\mathrm{BC}$ in idle time slot. And the channel is remained by the terminal that occupies it before in busy time slot.

3) Directional antenna-based mechanism, such as DMAC protocol. It is developed from IEEE 802.11. The protocol requires each node to know the location of themselves and their neighbors. Source node first launches a RTS/CTS/ACK handshake mechanism before sending data packets. RTS or Omni are sent directionally according to the situation of the transmission around. The directional antenna will be blocked when receiving the RTS or CTS, and the source nodes delay sending packets according to the received information. The conflict of transmission can be reduced by using directional antennas and channel reusing rate can be increased. But the directional antenna systems are expensive, and it's hard to maintain in the actual implementation.

TDMA-based MAC protocols require strict time synchronization and its slot is fixed. When a node sends no data, its slots cannot be occupied by other nodes that need to send data. So it can't use channel resources effectively. For example, in Japan ALOHA is mainly used in MAC layer protocol of Dedicated Short Range Communication (DSRC) [7]. It is designed for the electronic toll collection and other applications, and it can't meet the demands in many service application scenarios and services.

CSMA-based protocol does not require time synchronization. It uses carrier sense method to judge whether the channel is idle before sending data. If the channel is busy, it adopts backoff mechanism to make the node return to sense, and tries again. So the conflict probability is small and the channel utilization is effective. CSMAbased protocol is more suitable for the rapid movement of vehicles. Therefore, this paper focuses on the MAC protocol based on CSMA/CD mechanism.

\section{Conventional EDCA Mechanisms and Support of QoS}

QoS refers to a series of performance indexes that need to be satisfied when the network transmits some data flows. QoS guarantee reflects the capabilities that network devices or protocols ensure data transmission and meet specific service. It is described by the throughput, delay, delay jitter and packet loss rate and other parameters. In order to support the application of the Intelligent Transportation System, IEEE 802.11 Working Group established wireless access standard for the vehicle environment, named IEEE $802.11 \mathrm{p}$. In this standard, the MAC layer adopted the EDCA mechanism based on CSMA/CD.

The working principle of EDCA mechanism is shown in Figure 1.

EDCA provides the priority-based QoS function. This mechanism defines the access category (AC). Reference to $802.1 \mathrm{D}$ standard, it casts 8 priorities into $4 \mathrm{ACs}$, each of which has a separate backoff parameter. Hence, there are 4 separate competition entities in each node. The distinction between priorities is obtained by setting the values of different parameters such as Arbitration Inter Frame Space (AIFS), minimum contention window (CWmin) and maximum contention window (CWmax) are used for different ACs. For each $A C[i](i=1,2,3)$, the backoff mechanisms are the same as DCF. To achieve the distinction between priorities, for $0 \leq i<j \leq 3$, demand $C W_{\min }[i] \geq C W_{\min }[j], C W_{\max }[i] \geq C W_{\max }[j]$ and $A I F S[i] \geq$ $A I F S[j]$. As can be seen, higher-priorities have smaller CWmin, CWmax, AIFS parameters. They can enter the backoff process and finish it firstly in the competition. Thus they have a top priority to access to channel. If several entities finish backoff at the same time, the higher-priority entity will be sent as a priority.

Furthermore, the EDCA mechanism introduces the concept of transmission opportunity (TXOP). After AC competing for channels and having sent a frame successfully, 


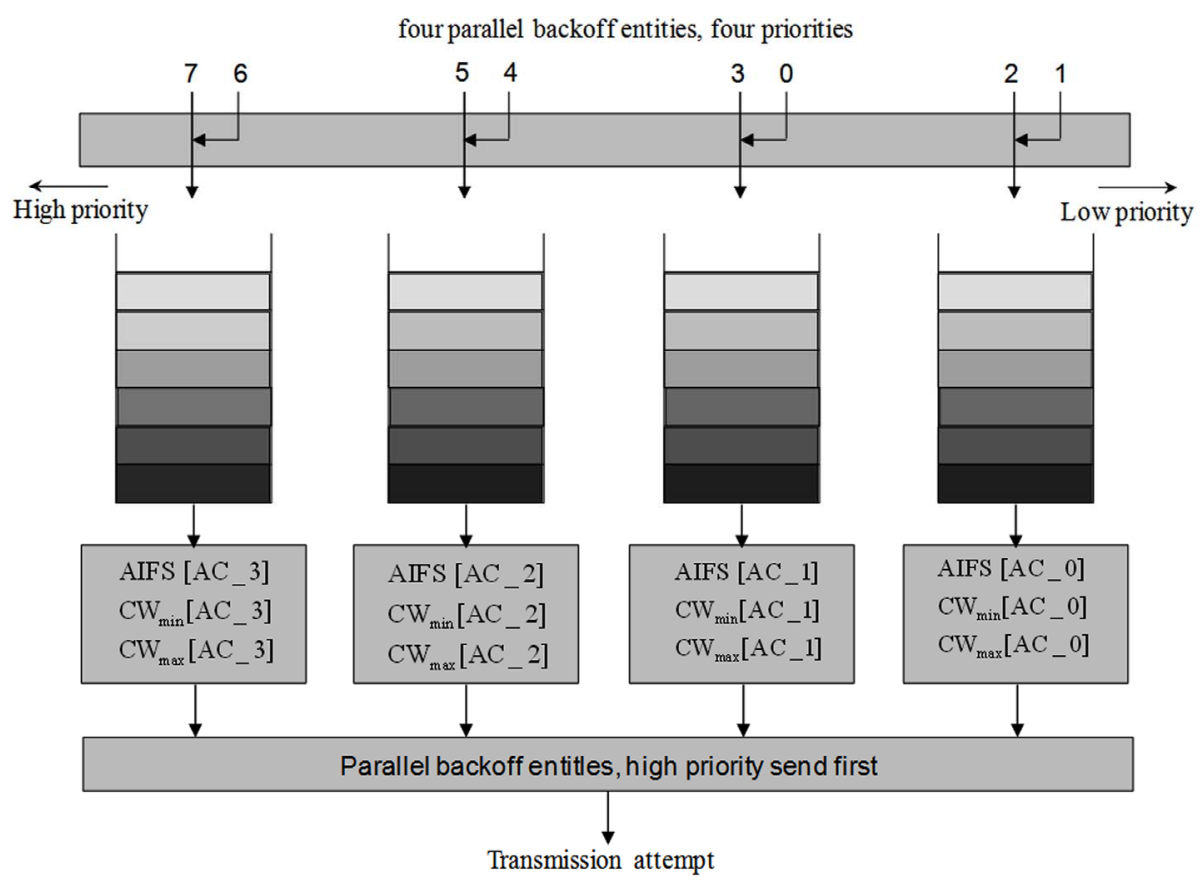

Figure 1. The working principle of EDCA mechanism

if there are still data frames need to be sent, the channel can be used all the time in the TXOP time limit. When we reach the TXOP time limit or there are no data frames to send, TXOP is over and starts a new competition.

The EDCA mechanism ensures the higher-priority service to be sent as a priority by the means of distinguishing priorities. However, in high load, for middle and low-priority service flows, the performance will become rather poor. For EDCA mechanism, parameters configuration is the key of effectively using channel resources. And the reasonable parameter adjustment algorithm plays an important role in expressing the system performance.

\section{New Design of Differentiation Enhanced Adaptive EDCA}

On one hand, the EDCA mechanism supports to send the high priority service firstly, but backoff parameters can not be adjusted dynamically with the change of network situation. Thus the network performance can't be utilized effectively. On the other hand, EDCA can distinguish competition parameters from different ACs that belong to the same node. It leads to data frames from the same AC of multiple sites choosing the same competition parameters, which tries to send at the same time, especially for the high priority AC with the same AIFS, its scope of the $\mathrm{CW}$ is small, thus the probability of choosing the same parameters is very large. This phenomenon is even more evident with the nodes increasing. For the above two aspects, this paper will design a Differentiation Enhanced
Adaptive EDCA (DE-AEDCA). The mechanism can adapt to different network congestions by calculating the collision rate of the sending-data frames, dynamically adjusting the window zooming, maintaining an appropriate contention window range. While the random offset achieves the further distinction of data frames competition parameters between the same priority, and reduces their conflict probability. Consequently, it cuts down the numbers of idle time slots caused by conflict, and improves the channel utilization.

\subsection{Selection of Determining the Network Status Parameters}

In the successive, same interval of time period $T_{n} \quad n=$ $(1,2,3, \cdots)$, we record the collision rate of different $A C[i]$ $(i=1,2,3)$ access types periodic. We assume the number of data frame conflicts to be $N_{n} m_{n}^{\text {collision }}[i]$ in one $T_{n}$, using parameter $P_{n}[i]$ to show the conflicts in the current time period $T_{n}$. The range of the $P_{n}[i]$ value is 0 to 1. The formula is shown in (1).

$$
P_{n}[i]=\operatorname{Num}_{n}^{\text {collision }}[i] / \operatorname{Num}_{n}^{\text {total }}[i] \quad(n=1,2,3, \cdots)
$$

Collision rate changes in each time period $T_{n}$ dynamically, which $T_{n}$ is an integer multiple of slot duration. If $T_{n}$ is too large, it cannot reflect real-time network status. On the contrary, if $T_{n}$ is too small, the computation will increase. When OFDM technology is adopted in 
the physical layer, one time slot length is $9 \mu \mathrm{s}$, data interval is $0.01 \mathrm{~s}$ in this article. Then we take the time data sources to produce 9 data frames as a statistical cycle, which means $T_{n}=90000 \mu \mathrm{s}$. In order to suppress judging errors of network state due to the burst of data frame, we deal with the current $P_{n}[i]$ with weighted average method of multiple time periods collision rate. Weights after treatment is $P_{n}^{\text {weight }}[i]$, which is computed as (2):

$$
\begin{cases}P_{n}^{\text {weight }}[i]=k \times P_{n}[i]+(1-k) \times P_{n-1}^{\text {weight }}[i], & (n \geq 2) \\ P_{n}^{\text {weight }}[i]=P_{n}[i], & (n=1)\end{cases}
$$

where $k(0 \leq k \leq 1)$ is the smoothness factor. It is changed by the emergent state of network, for instance, estimated by comparing $N_{u m}^{\text {total }}[i]$ with $N u m_{n-1}^{\text {total }}[i]$. If the nodes generate stable value of data frames in each time period, $k$ can take a higher value. In this paper, it is more stable to produce data of source node. According to experimental results, $k$ takes 0.8 in the simulation of this paper.

\subsection{Conflict Process of Data Frame}

The algorithm for conflict process of data frame is given as:

$$
\begin{aligned}
& \left\{\begin{array}{l}
C W_{\text {new }}[i]=\min \left\{C W_{\max }[i], C W_{\text {old }}[i] \times\left(1+2^{\varphi}\right)\right\}, \\
\varphi=P_{n}^{\text {weight }}[i],
\end{array}\right. \\
& (i=0,1,2,3)
\end{aligned}
$$

where $\varphi$ is the current weighted value of collision rate, $\varphi \in[0,1]$. The scope of $1+2^{\varphi}$ is $[2,3]$, thus the competition window of $A C[i]$ can progressively increase in different multiples depending on collision rate of its data frame. The larger the value of $P_{n}^{\text {weight }}[i]$ is, the greater the progressively increasing multiples is, and vice versa, thus competition window can adaptively adjust depending on network status indication.

In this algorithm, each access category $P_{n}^{\text {weight }}[i]$ is statistical respectively, because the high-priority data has higher right to access to channel than that of the lowpriority data, so $P_{n}^{\text {weight }}[i] \leq P_{n}^{\text {weight }}[j],(0 \leq i \leq j \leq 3)$, it means that the higher the priority is, the smaller collision rate is. Thus the progressively increasing multiples of their competition window are smaller. Therefore, this algorithm still strictly ensure the priority relations among all categories of data, it is critical for the application of intelligent transportation system, because it must ensure that high-priority data (such as emergency short message data) to send first.

\subsection{Judgement of Sending the Data Frames Successfully}

The equations are an exception to the prescribed specifi- cations of this template. You will need to determine whether or not your equation should be typed using either the Times New Roman or the Symbol font (please no other font). To create multileveled equations, it may be necessary to treat the equation as a graphic and insert it into the text after your paper is styled.

First, we define a variable $\gamma_{i}$ whose value shows the current consecutively sending number of data frame of node $A C[i]$. Its value adds 1 when it successfully sends a data frame, and the value is cleared when a conflict occurred.

Secondly, we subtract the value $C W_{\min }[i] \times\left(1-P_{n}^{\text {weight }}[i]\right)$ of the contention window when $\gamma_{i}=1$. Subtract $2 \times$ $C W_{\min }[i] \times\left(1-P_{n}^{\text {weight }}[i]\right)$ of the contention window when $\gamma_{i}=2$, and so on. That means the decreasing value increases when $\gamma_{i}$ increases every time. Every time, the increasing value is $\Delta$ :

$$
\Delta=C W_{\min }[i] \times\left(1-P_{n}^{\text {weight }}[i]\right)
$$

Finally, when the value of contention window reduced to half of the initial value, it achieves the congestion avoidance phase, in order to avoid excessive competition which results from contention window decreasing too fast. Hereafter, when $\gamma_{i}$ keeps on increasing, it resumes taking $C W_{\min }[i] \times\left(1-P_{n}^{\text {weight }}[i]\right)$ as original decreasing value and beginning to accelerate declining as beforementioned steps until declining to $C W_{\min }[i]$.

The basic idea of this algorithm is similar to slow-start decreasing algorithm SSDS (Slow-start Decrease Scheme. But it increases the adaptive capacity of the decreasing window, and it adds congestion control to avoid further competition which results from contention window decreasing too fast. The range of $\left(1-P_{n}^{\text {weight }}[i]\right)$ is $[0,1]$ in the algorithm, and the range of $\Delta$ is $\left[0, C W_{\min }[i]\right]$. The larger $P_{n}^{\text {weight }}[i]$ is, the more severe the current network congestion is, and the smaller $\Delta$ is, the slower the declining pace of contention window is, and vice versa. The algorithm can judge the current network state and adjust the contention window size dynamically, and maintain a good state of congestion avoidance.

The algorithm can be described as (7):

$\left\{\begin{array}{l}C W_{\gamma_{i}}[i]=\max \left\{C W_{\min }[i], C W_{\gamma_{i}-1}[i]-\Delta \times \gamma_{i}\right\}, \quad\left(\gamma_{i} \leq m\right) \\ C W_{\gamma_{i}}[i]=\max \left\{C W_{\min }[i], C W_{\gamma_{i}-1}[i]-\Delta \times\left(\gamma_{i}-m\right)\right\}, \quad\left(\gamma_{i}>m\right)\end{array}\right.$

where $\gamma_{i}$ is the number of sending data frame successfully after the congestion avoidance phase, and $C W_{\gamma_{i}}[i]$ is the value of the contention window which is calculated by the algorithm. For example, $C W_{0}[i]$ shows the initial 
contention window before the first time successfully sending data frame. $m$ means the number of sending data frames continuous successful before the congestion avoidance phase.

$$
\left\{\begin{array}{l}
C W_{m}[i] \geq \frac{1}{2} C W_{0}[i], \\
C W_{m+1}[i]<\frac{1}{2} C W_{0}[i],
\end{array} \quad m \in \gamma_{i}\right.
$$

\subsection{Discrimination of Same Access Categories}

Another improvement in this paper is the differentiation enhancement. It is designed to resolve the problem that the same AC data frames of different nodes compete with each other leads to the drastic decline of the whole network's performance when EDCA is under the high load.

Usually, the waiting time before trying to transfer each $\mathrm{AC}$ data frame equals to the sum of the avoidance time and the delay time. The avoidance time is random generated between 0 and $C W[i]$. The delay time which is decided by $A I F S[i]$ is the time necessary to wait before avoidance. So the basic idea of this algorithm is to increase the probability of the same AC choosing different competition parameter value by designing an added random offset to AIFS[i] and $C W[i]$, on this condition, it's easier to distinguishing the competition parameters of the same AC data frames of different nodes. The concrete processes of the algorithm are as follows:

- The CW-based offset

The biggest offset $C W_{\text {offset }}^{\max }[i]$ is computed by

$$
\left\{\begin{array}{l}
C W_{\text {offset }}^{\max }[i]=\left[\left(1+C W_{\min }[i]\right)^{\varphi}\right], \\
\varphi=P_{n}^{\text {weight }}[i],
\end{array} \quad(i=0,1,2,3) .\right.
$$

Then a random value between 0 and $C W_{\text {offset }}^{\max }[i]$ is selected as the real offset by

$$
C W_{\text {offset }}^{\max }[i]=\operatorname{Random}\left(0, C W_{\text {offset }}^{\max }[i]\right), \quad(i=0,1,2,3) .
$$

Finally, according to (11), the real avoidance time can be computed as

$$
\begin{aligned}
& T= {[\operatorname{random}(0, C W[i])} \\
&\left.+C W_{\text {offset }}[i] \times \text { aSlotTime }\right], \\
&(i=0,1,2,3)
\end{aligned}
$$

Equations (10) and (11) show that the random offset changes with the state of the whole network. The larger the value of network congestion condition is, the larger $C W_{\text {offset }}^{\max }[i]$ is, it means the larger the choice scope of the offset is, the smaller the probability of choosing the same offset is.

- AIFS-based offset

Above all, this algorithm can further distinguish the same AC by AIFS offset. First of all, assign an offset scope $\left[N_{i}, M_{i}\right]$ for each $\mathrm{AC}$ by its priority. Random select an integer as the number of slot time of AIFS[i].

$$
\operatorname{AIFS}_{\text {offset }}[i]=\operatorname{Random}\left[N_{i}, M_{i}\right] \times \text { aSlotTime }
$$

Secondly, we put it as offset and add it to AIFS[i], then computing the $\operatorname{AIFS}_{\text {new }}[i]$.

$$
\operatorname{AIFS}_{\text {new }}[i]=A I F S[i]+\operatorname{AIFS}_{\text {offset }}[i]
$$

Though the introduction of the offset, we decline the probability of the same AC data frame choosing the same competition parameter and reduce the collision rate between them, which is in favor of improving the performance of the whole network.

The flow chart of the algorithm is shown in Figure 2.

\section{Simulation Studies and Analysis}

The simulation experiments are conducted on OPNET platform. The OPNET uses process domain model, node domain model and network domain model to react the relevant features of the networks comprehensively. Finitestate machines, OPNET core function, and standard $\mathrm{C}$ and $\mathrm{C}++$ can make up the process model to describe bottom layer algorithm and the pipe stage model. OPNET Modeler uses discrete-time driven simulation mechanism that can get very detailed simulation results. It greatly improves the efficiency of the simulation.

\subsection{Design of Simulation Cases}

The simulation in this paper only considers the impact of MAC layer protocol on QoS requirements of multiple intelligent transportation service. Meanwhile, to cast the impact of routing layer on the wireless communication performance, this paper only considers one-leap communication without considering the impact on wireless communication of barrier and relative speed. The results in [11] shows that in one-hop range, the impact on throughput and delay of distance and speed of the cars is not obvious, but it has much relation with the number of the vehicles communicating at the same time. This paper mainly simulates different cases by setting different numbers of vehicles. In the simulation, the service scenario can be divided into high density case, middle density case and low density case by the number of vehicles communicating at the same time.

\subsection{Parameter Settings}

The services in Intelligent transportation systems can be divided into four categories: active safety, such as warning of dangerous road, collision warning; public service, such as emergency vehicles (fire engine, ambulance) which can pass prior on congested road, emergency speech communications; driving assistance service, such as roads na vigation, video monitoring; business and entertainment 


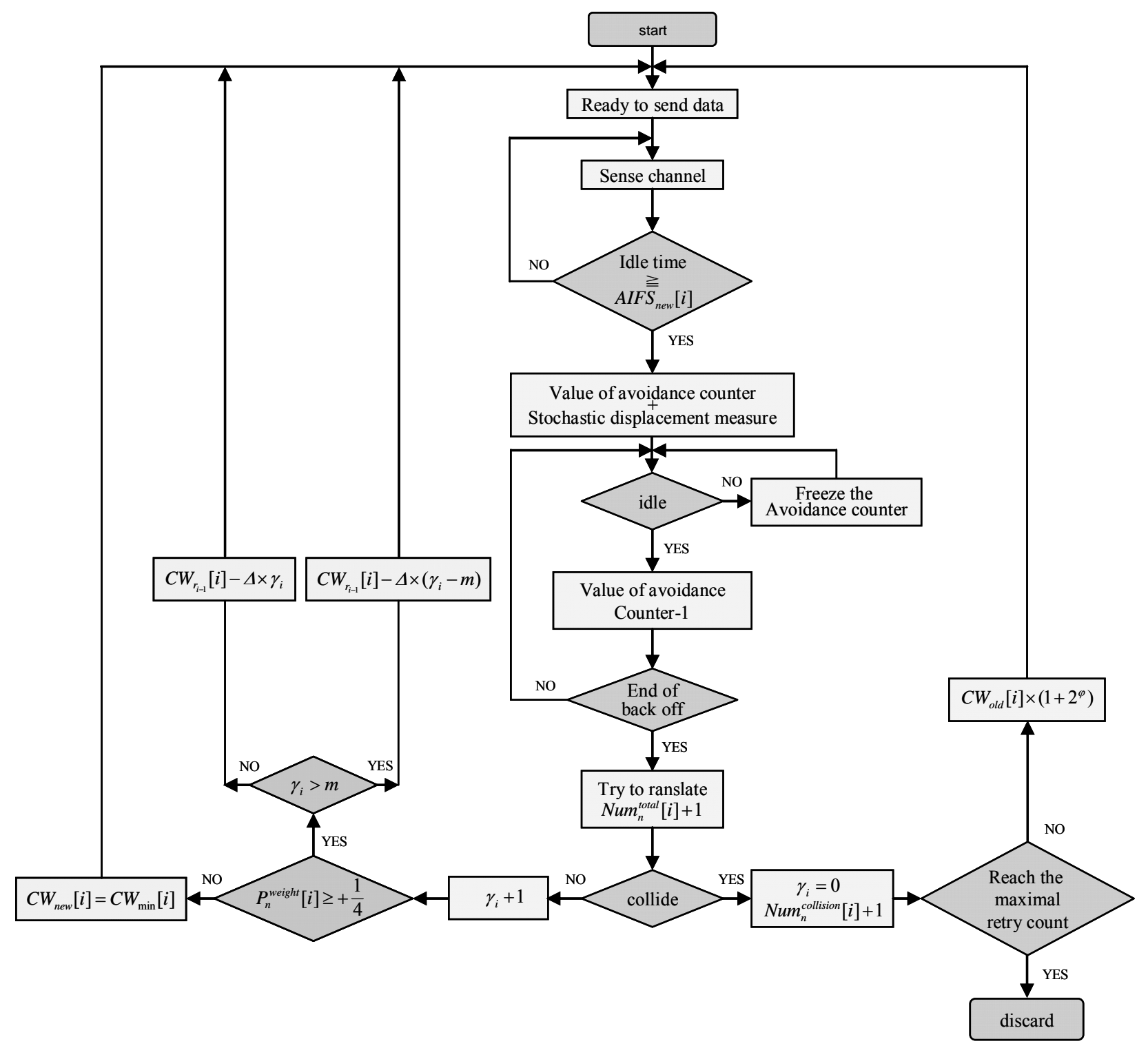

Figure 2. The flow chart of the differentiation enhanced adaptive EDCA

service, such as Internet access, electronic map download.

In view of the above service categories, application-layer sets four services in the simulation: emergency short message, speech, video, background, and the priority are from high to low. The service parameters are in Table 1. This paper only considers the MAC layer's effects for QoS, neglect routing layer and the above ones. The high layer is simulated by sending source modules.

Physical layer uses OFDM modulation technique, its bandwidth is $20 \mathrm{MHz}$, transmission rate is $24 \mathrm{Mbits} / \mathrm{s}$, SIFS is 16 us, DIFS is 34 us, and a SlotTime is 9 us. It distinguishes the different cases by setting different numbers of vehicle nodes. 32 nodes are set in high-density vehicle case; 20 nodes are set in middle-density vehicle case; and 12 nodes are set in low-density vehicle case. In each case every vehicle node carries only one service, and in different cases the numbers of vehicle nodes which carry different services are same. The parameters of

Table 1. Parameter list

\begin{tabular}{cccc}
\hline service & $\begin{array}{c}\text { Inter-packet } \\
\text { Gap(s) }\end{array}$ & $\begin{array}{c}\text { Packet } \\
\text { size }\end{array}$ & $\begin{array}{c}\text { Transmission rate } \\
(\text { Kbits/s) }\end{array}$ \\
\hline emergence & 0.01 & 12 & 9.6 \\
speech & 0.01 & 80 & 64 \\
video & 0.01 & 1250 & 1000 \\
background & 0.01 & 1500 & 1200 \\
\hline
\end{tabular}


MAC layer are showed in Table 2, $A C[3], A C[2], A C[1]$, $A C[0]$ are emergency short message, speech, video, background services.

The AIFSN offset range of the mechanism proposed in this paper is in Table 3.

\subsection{Simulation Results}

In the simulation, the performance of network throughput and delay are analyzed. Network throughput refers to the summation of data packets received and successfully transmitted to application layer by MAC layer in all sites. Delay means different packets start when sending node in the application layer generates and end when destination node receives those packets, including media access delay and transmission delay (ms).

Figures 3, 4 and 5 are the simulation results with high density, medium density, and low density, respectively. The corresponding values of the four data streams in EDCA mechanism are described as the curves with round, left triangle, upper triangle and square symbols, according to their descending priority. The corresponding values of the four data streams in DE-AEDCA mechanism are described as the curves with the plus sign, lower triangle, right triangle and diamond symbols, by their priority from high to low. In figures, the $\mathrm{x}$-axis represents the simulation time (sec.), the y-axis represents the delay time (sec.) and throughput (bit/s) separately. Table 4 is the statistic results for the three types of cases in simulation.

The simulation results with high-density of vehicles, show that, in comparison to conventional EDCA, the delays of various services in the proposed DE-AEDCA mechanism are decreasing. Thus, it can meet the demands of security-related emergency short messages and QoS requirements of speech service. Meanwhile the throughput of video service increases from $6100 \mathrm{kbits} / \mathrm{s}$ up to 8000 $\mathrm{kbits} / \mathrm{s}$, and the packet loss rate drops to $6.25 \%$. In addition, the throughput of background service rises from

Table 2. Mac layer parameter

\begin{tabular}{cccc}
\hline & $\mathrm{CW}_{\text {min }}$ & $\mathrm{CW}_{\max }$ & AIFS(us) \\
\hline $\mathrm{AC}[3]$ & 7 & 15 & 34 \\
$\mathrm{AC}[2]$ & 15 & 31 & 34 \\
$\mathrm{AC}[1]$ & 31 & 1023 & 52 \\
$\mathrm{AC}[0]$ & 31 & 1023 & 79 \\
\hline
\end{tabular}

Table 3. Aifsn offset range of different Ac

\begin{tabular}{ccc}
\hline & $\mathrm{Ni}$ & $\mathrm{Mi}$ \\
\hline $\mathrm{AC}[3]$ & 0 & 6 \\
$\mathrm{AC}[2]$ & 0 & 6 \\
$\mathrm{AC}[1]$ & 3 & 9 \\
$\mathrm{AC}[0]$ & 3 & 9 \\
\hline
\end{tabular}

$1850 \mathrm{kbits} / \mathrm{s}$ to $3250 \mathrm{kbits} / \mathrm{s}$. Thus throughput of the video service and the background service respectively increases by $31.1 \%$ and $75.7 \%$. Therefore the total throughput improves $38.6 \%$. To sum up, the overall performances are improved.

For the middle-density case, the delay of various services has a certain decrease; the bandwidth needs of background service are met; no packet loss occurs; and the throughput performance is improved. In comparison to the EDCA mechanism, its overall performance is improved to some extent.

For the low-density vehicles, the delay is smaller than that in EDCA mechanism. Network packet loss does not occur any more. So the DE-AEDCA mechanism can meet the needs of various service applications properly.

As can be seen from the simulation results in the three situations of high-density and high network load, middledensity and high network load, as well as low-density and low network load, DE-AEDCA mechanism has both a higher network throughput and a smaller delay compared with EDCA mechanism. The reason is DE-AEDCA

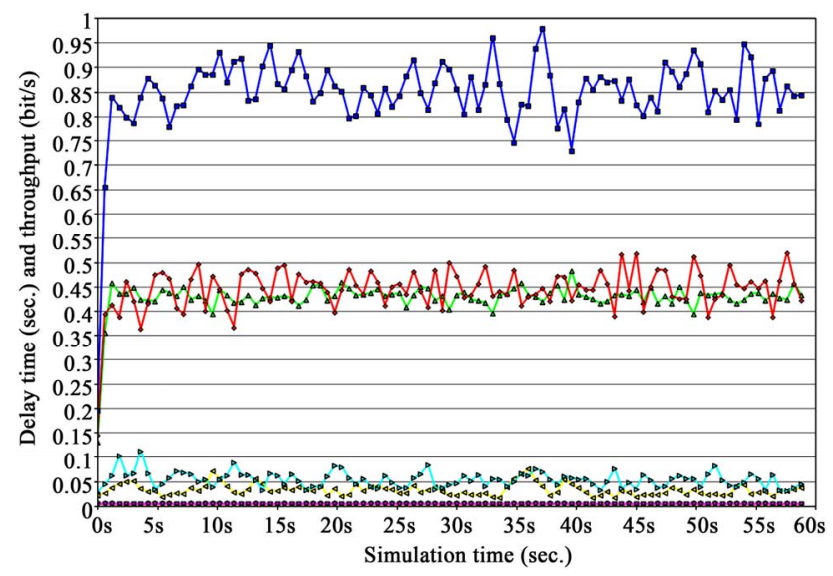

(a)

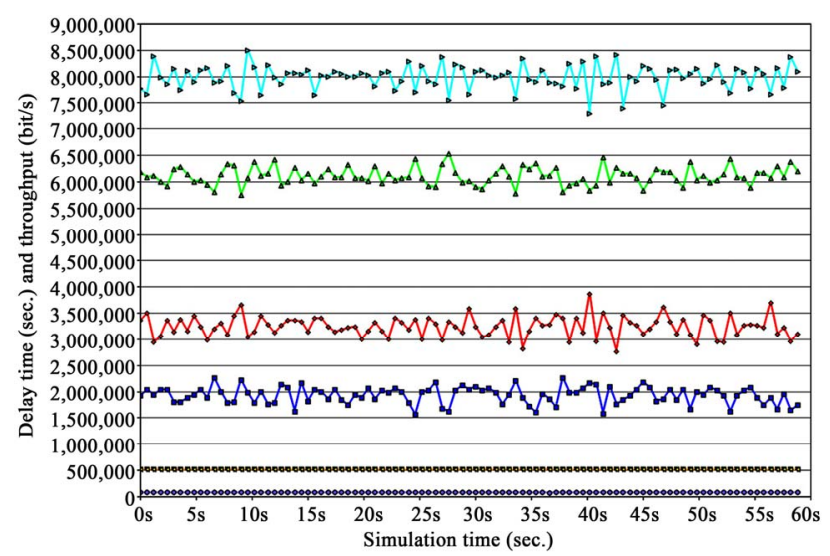

(b)

Figure 3. The simulate results at high density. (a) medium access delay; (b) network throughput 


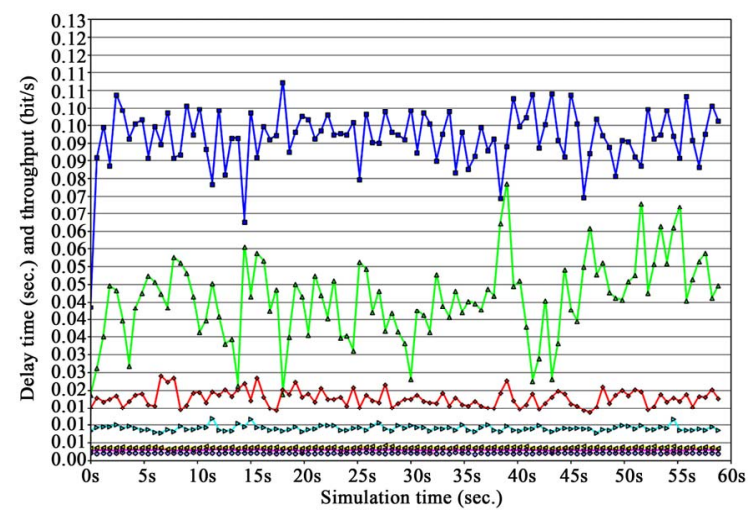

(a)

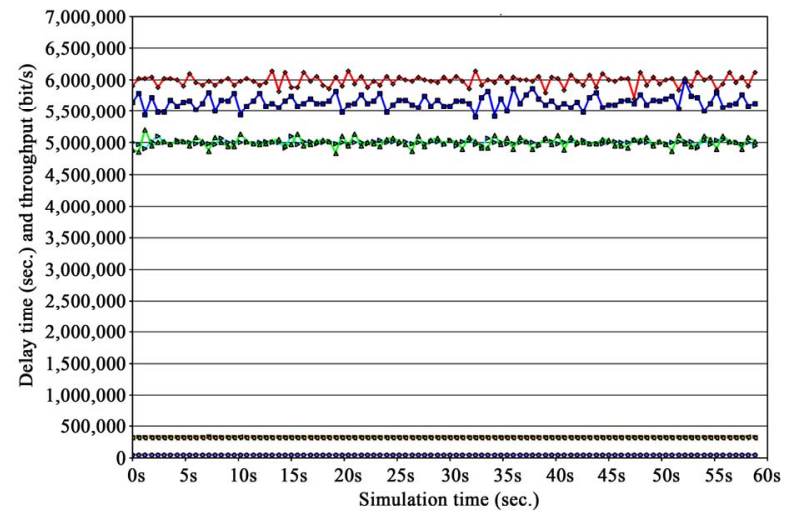

(b)

Figure 4. The simulate results at middle density. (a) medium access delay; (b) network throughput

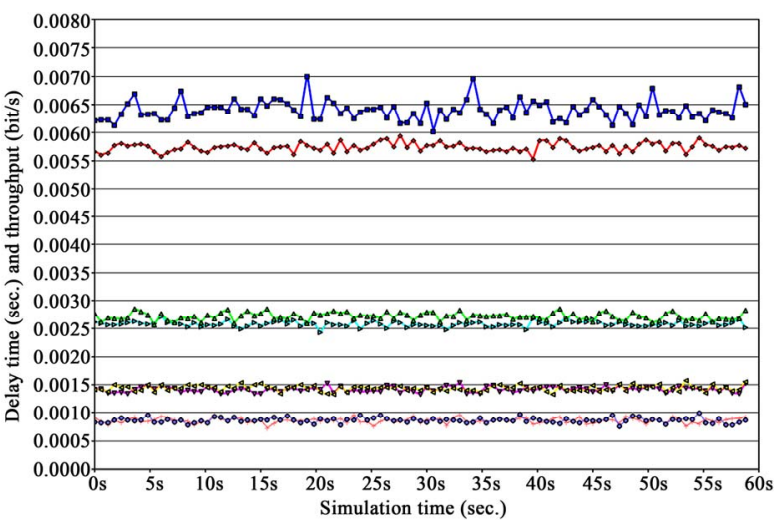

(a)

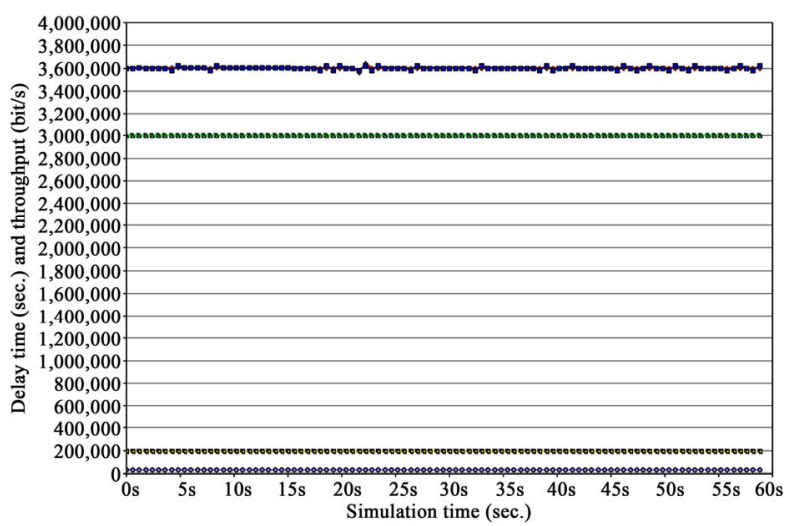

(b)

Figure 5. The simulate results at low density. (a) medium access delay; (b) network throughput

Table 4. The statistical results of simulations

\begin{tabular}{|c|c|c|c|c|c|c|c|c|c|}
\hline & & \multicolumn{4}{|c|}{ EDCA } & \multicolumn{4}{|c|}{ DE-AEDCA } \\
\hline & & $\mathrm{AC}[3]$ & $\mathrm{AC}[2]$ & $\mathrm{AC}[1]$ & $\mathrm{AC}[0]$ & $\mathrm{AC}[3]$ & $\mathrm{AC}[2]$ & $\mathrm{AC}[1]$ & $\mathrm{AC}[0]$ \\
\hline \multirow{3}{*}{$\begin{array}{c}\text { Delay/ } \\
\text { average } \\
(\mathrm{ms})\end{array}$} & high & 6 & 34 & 430 & 850 & 2.5 & 4.6 & 55 & 450 \\
\hline & middle & 2.1 & 3.7 & 43 & 91 & 1.9 & 2.8 & 9 & 18 \\
\hline & low & 1.1 & 1.7 & 2.8 & 6.6 & 0.9 & 1.4 & 2.6 & 5.7 \\
\hline \multirow{3}{*}{$\begin{array}{c}\text { Throughput/ } \\
\text { average } \\
\text { (Kbits/s) }\end{array}$} & high & 76.8 & 512 & 6100 & 1850 & 76.8 & 512 & 8000 & 3250 \\
\hline & middle & 48 & 320 & 5000 & 5600 & 48 & 320 & 5000 & 6000 \\
\hline & low & 28.8 & 192 & 3000 & 3600 & 28.8 & 192 & 3000 & 3600 \\
\hline
\end{tabular}

mechanism considers the adjustment of competition parameters in conditions of a low network load and a high network load, so whenever in high-density and middledensity, or in the low-density vehicle cases, DE-AEDCA mechanism can have better QoS performance than EDCA mechanism.

In addition, among the data flows of same priority,
DE-AEDCA mechanism increases the range of the parameters and reduces the possibility of choosing the same parameter by choosing a random offset, thus it can reduce conflict of the same priority data in network and improve the channel utilization. Experiments show that the DE-AEDCA mechanism always has better performance than EDCA mechanism in all kinds of intelligent 
service case.

\section{Conclusions}

This paper considers QoS demands of various services in intelligence transportation, and analyzes the QoS based on the EDCA mechanism in IEEE 802.11p standard framework. A Differentiation Enhanced Adaptive EDCA is designed, which improves the QOS of EDCA by dynamically adjusting the size of competition window and further distinguishing the competition parameters of the data frames that have the same priority. The simulation results show that, in different situations of network congestion, the mechanism can discriminate the service priority, and make the high-priority service with low delay and high throughput. Meanwhile the overall performance of the network is improved significantly. Its performance is better than EDCA.

\section{Acknowledgements}

This work is supported by the National High Technology Research and Development Program of China (863 Program) (No. 2007AA11Z227).

\section{REFERENCES}

[1] T. Yashiro, T. Kondo, H. Yagome, M. Higuchi and Y. Matsushita, "A Network Based on Inter-Vehicle Communication," Proceedings of Intelligent Vehicles Symposium, 14-16 July 1993, pp. 345-350.

[2] H. Fujii, O. Hayashi and N. Nakagata, "Experimental Research on Inter-Vehicle Communication Using Infrared
Rays," Proceedings of IEEE Intelligent Vehicles Symposium, Tokyo, 19-20 September 1996, pp. 266-271.

[3] IEEE P802.11pTM/D6.0 Draft Standard for Information Technology Telecommunications and information exchange between Systems Local and metropolitan area networks-Specific requirements, Part 11: Wireless LAN Medium Access Control and Physical Layer specifications, November 2008.

[4] F. Borgonovo, A. Capone, M. Cesana and L. Fratta, "ADHOC MAC: New MAC Architecture for Ad Hoc Networks Providing Efficient and Reliable Point-to-Point and Broadcast Services," Wireless Networks, Vol. 10, No. 4, July 2004, pp. 359-366.

[5] Y. Ko, V. Shankarkumar and N. Vaidya, "Medium Access Control Protocols Using Directional Antennas in Ad Hoc Networks," Proceedings of IEEE INFOCOM 2000, Tel Aviv, Vol. 1, March 2000, pp. 13-21.

[6] K. Fujimura and T. Hasegawa, "A Collaborative MAC Protocol for Inter-Vehicle and Road to Vehicle Communications," Proceddings of IEEE Intelligent Transportation Systems Conference 2004, Washington, D.C., October 2004, pp. 816-822.

[7] "Standard Specification for Telecomunications and Information Exchange between Roadside and Vehicle Systems $-5 \mathrm{GHz}$ Band Dedicated Short Range Communications (DSRC) Medium Access Control (MAC) and Physical Layer (PHY),”ASTM E2213-03, September 2003.

[8] J. Wang, S. Makfile and J. Li, "A Random Adaptive Method to Adjust MAC Parameters in IEEE802.11e WLAN," Journal of Central South University of Technology, Vol. 16, pp. 629-634, 2009. 